\title{
Racial disparities in psychological distress in post-apartheid South Africa: results from the SANHANES-1 survey
}

\author{
Nigel Walsh Harriman ${ }^{1}(1) \cdot$ David R. Williams ${ }^{1,2} \cdot$ Justin Winston Morgan ${ }^{1} \cdot$ Ronel Sewpaul $^{3} \cdot$ Thabang Manyaapelo $^{3}$. \\ Sibusiso Sifunda ${ }^{3}$. Musawenkosi Mabaso ${ }^{3}$ - Anthony David Mbewu ${ }^{4} \cdot$ Sasiragha Priscilla Reddy M $^{3,5}$
}

Received: 14 May 2021 / Accepted: 30 August 2021 / Published online: 6 October 2021

(c) Springer-Verlag GmbH Germany, part of Springer Nature 2021

\begin{abstract}
Purpose South Africa has long endured a high prevalence of mental disorders at the national level, and its unique social and historical context could be a contributor to an increased risk of mental health problems. Our current understanding is limited regarding the relative importance of various social determinants to mental health challenges in South Africa, and how existing racial inequities may be explained by these determinants.

Methods This study attempted to elucidate potential social determinants of mental health in South Africa using data from the nationally representative South African National Health and Nutrition Examination Survey (SANHANES-1). The main outcome of interest was psychological distress, measured with the Kessler-10 scale. Hierarchical linear regression models included covariates for demographic and socioeconomic factors, count of traumatic events, and a series of stress-related constructs. Analyses were conducted on two populations: the entire sample $(n=15,981)$, and the African subpopulation $(n=10,723)$.

Results Regression models on the entire sample indicated racial disparities in psychological distress, with Africans experiencing higher distress than White and Coloured individuals. Results within the African sub-population indicated geo-spatial disparities, with Africans in formal urban settings experiencing higher psychological distress than those living in formal and informal rural locales. Across both samples, results indicated a cumulative association between count of stressors and traumatic events and distress.

Conclusion We found racial disparities across several mental health-related domains. Africans had greater exposure to traumatic events, social stressors, and psychological distress. This research is a necessary foundation for public health interventions and policy change to effectively reduce inequities in psychological distress.
\end{abstract}

Keywords Psychological distress · Racial disparities · Stressors · Traumatic events · South Africa

Nigel Walsh Harriman

nharriman@hsph.harvard.edu

David R. Williams

dwilliam@hsph.harvard.edu

Justin Winston Morgan

jmorgan@g.harvard.edu

Ronel Sewpaul

rsewpaul@hsrc.ac.za

Thabang Manyaapelo

tmanyaapelo@hsrc.ac.za

Sibusiso Sifunda

ssifunda@hsrc.ac.za

Musawenkosi Mabaso

mmabaso@hsrc.ac.za

Anthony David Mbewu

tonymbewu@gmail.com
Sasiragha Priscilla Reddy

preddy@hsrc.ac.za

1 Department of Social and Behavioral Sciences, Harvard T.H. Chan School of Public Health, 677 Huntington Avenue, Kresge Building, Boston, MA 02115, USA

2 Department of African and African American Studies, Harvard University, 12 Quincy st, Cambridge, MA 02138, USA

3 Human and Social Capabilities (HSC) Department, Human Sciences Research Council (HSRC), Private Bag X9182, Cape Town 8000, South Africa

4 School of Medicine, Sefako Makgatho Health Sciences University, Molotlegi Street, Ga-Rankuwa, Pretoria, South Africa

5 Faculty of Health Sciences, Nelson Mandela University, PO Box 77000, Port Elizabeth 6031, South Africa 


\section{Introduction}

South Africa has long endured a high prevalence of mental disorders. The 2004 South African Stress and Health (SASH) study found that its lifetime prevalence of anxiety and mood disorders was nearly three times that of Nigeria, the only other African country surveyed [1]. The prevalence of any mental disorder was also higher than other low-middle income countries, such as China, Lebanon, and Mexico [2-4].

South Africa's unique social and historical context may contribute to an increased risk of mental health problems. Social and economic inequities that were created by Apartheid-era colonialism and institutional racism persist in contemporary society, with striking inequities present along racial lines [5-8]. South Africa is one of the most unequal societies in the world, as racial disparities exist in housing, healthcare access, health, and multiple indicators of socioeconomic status (SES), all of which can contribute to an increased risk of poor mental health [9-12]. Indeed, a strong consensus in the stress literature indicates that socio-contextual factors play a role in shaping population mental health risks, especially those factors that reflect exposure to chronic and acute stressors linked to living and working conditions [13, 14]. For example, studies using the Pearlin's Stress Process Model have documented a negative association between social stressors and mental health, especially in low income and marginalized populations [15-17]. While prior research has documented the prevalence of mental health disorders in South Africa, the contextual determinants of mental health, especially those linked to racial inequities, or social conditions that might be amenable to clinical or policy interventions, have received limited research attention.

Psychological distress is an aspect of mental health that has received increasing research attention in the South African context. Many studies investigating psychological distress utilize the Kessler-10 and Kessler-6, brief measures that demonstrate acceptable psychometric properties and have been shown to predict both depression and anxiety disorders within the South African general population [18-21]. Further research is needed into how specific conditions of life captured by stressors differentially influence psychological distress. This empirical understanding is a necessary foundation for public health interventions to effectively reduce inequities in psychological distress.

To our knowledge, there have been three nationally representative probability surveys that include measures of psychological distress in South Africa since the end of Apartheid. Conducted between 2002 and 2004, the SASH study examined social correlates of psychological distress, particularly those captured by specific stressors related to social context $[22,23]$ This study found that cumulative exposure to multiple traumatic events, including racialpolitical violence, was linked to increased distress [24]. Other analyses of these data indicated that there were racial inequities in psychological distress; with African, Coloured, and Indian subpopulations all reporting greater psychological distress than Whites. Such differences were partially explained by social stressors, including stressful life events and unfair treatment [25]. Another finding from the SASH study revealed that social stressors contributed to explaining racial differences in self-esteem and mastery - the degree to which individuals perceive they can control their life situation [26, 27]. Since the findings from the SASH study, no other nationally representative studies have examined the association between social conditions captured by stressors and mental health outcomes.

The National HIV Prevalence, Incidence, and Behavior Survey, a longstanding, nationally representative survey aimed at investigating the epidemiology of HIV in South Africa, began to include questions about psychological distress in 2012 [28, 29]. Findings revealed that Africans had more than twice the odds of experiencing psychological distress compared to other racial groups, but the social conditions that could explain these inequities were not examined.

The South African National Health and Nutrition Examination Survey (SANHANES-1) is another nationally representative probability survey that examined the correlates of psychological distress [30]. Analyses of SANHANES-1 data have documented that distress is a risk factor for multiple health outcomes, including insomnia, World Health Organization Disability Assessment Schedule score, serum C-Reactive Protein, and BMI [31-34]. However, although the SANHANES-1 measures multiple contextual stressors, their relationship to psychological distress has yet to be investigated. Thus, the SANHANES data provide a unique opportunity to update and expand on the knowledge base established by the SASH, by providing a more recent assessment of the extent of racial inequities in psychological distress and the contribution of social determinants to these patterns.

Another critical component of South Africa's social context lies in the geospatial distribution of psychological distress among Africans, who constitute about $80 \%$ of the general population. Despite the heterogeneity amongst Africans in ethnicity, culture, language, and geographic location, prior research uses a relatively limited conceptualization of race, treating Africans as a homogenous racial group [29, 30]. Most existing research on psychological distress has compared Africans to other major racial groups, with scant attention given to how distress may vary within the African population. While some work has compared the correlates of poor mental health between historically disadvantaged urban and rural communities, this has yet to be examined 
nationally [35]. To our knowledge, the only nationally representative study that examines geographic differences in health amongst Africans found that urban youth were more likely to smoke, have high salt intake, be physically inactive, and be obese, compared to youth in rural geographies [36]. Examining geographic variation in psychological distress among Africans and how exposure to socio-contextual stressors influences that variation will allow future research to more critically assess how differential social conditions experienced by Africans may influence mental health.

To address the knowledge gaps regarding racial inequities in psychological distress, the role of social conditions as reflected in stressors contributing to racial inequities, and the extent to which the socio-geographic context of Africans is related to their risk of psychological distress, this study aims to:

(1) Assess the extent of racial disparities in psychological distress in a nationally representative population in South Africa

(2) Explore the relationship between psychological distress and the social conditions of life in South Africa as captured by stressors and traumas

(3) Examine the differences in psychological distress across formal/informal and urban/rural locales within the African subpopulation.

\section{Methods}

\section{Data}

This study analyzed data from the SANHANES-1, a crosssectional national survey conducted in 2011-2012 which included interviews and medical examinations with biomarker analyses [30]. Sample households were selected using a multi-stage, disproportionate, stratified cluster sampling approach, with 500 enumeration areas (EAs) selected based on province, geographic type, and race. A random sample of 20 households was selected from each of the enumeration areas, yielding a 10,000-household sample of eligible participants. At the household level, 8166 of the 10,000 households were occupied; these households yielded 27,580 individuals of all ages who were eligible to be interviewed and agreed to participate, 25,532 (92.6\%) of whom completed the interview.

\section{Variables}

The main outcome of interest was psychological distress, measured with the Kessler-10 [18, 37]. The K-10 was developed as part of the National Health Interview Survey (NHIS) to measure the frequency of non-specific anxiety and depressive symptoms experienced in the past month [37]. It has demonstrated adequate psychometric properties for predicting both depression and anxiety in South Africa [20, 21].

The analysis included covariates for demographics, SES, count of traumatic events, and a series of stress-related constructs. Race was reported as per Statistics South Africa's standard population groups: African, Coloured, White, and Indian. In the South African context, the term "Coloured" refers to a heterogenous racial group which primarily consists of persons of mixed racial ancestry. Marked racial stratification developed during the Apartheid era in South Africa placed Whites at the top, Indians and Coloureds in the middle, and Africans at the bottom [38]. Geographic location was categorized as formal urban, informal urban, formal rural, or informal rural [39-42]. Age was categorized into 10 -year groups. Education was partitioned into three categories: 'No Formal Education', 'Educated through grades 8-12', or 'Higher Education (with or without matriculation)'. Income was cut at intervals that correspond roughly to the expected exponential distribution of income. A wealth index was created by summing various household amenities and asset ownership, and this index was categorized into quintiles [43]. We included multiple indicators of stress-related constructs were used in the analysis. First,we included a variable that was a count of traumatic events, based on a checklist including, experiencing a violent personal assault, a severe automobile accident, or a natural disaster. Second, we included indicators for seven indicators of chronic stressors in the analysis: food insecurity (hunger-related stress), alcohol-related stress in the household, household crowding, neighborhood inaccessibility, economic stress, interpersonal conflict, and crime and alcohol-related stress in the neighborhood. Our food insecurity measure was based on the Community Childhood Hunger Identification Project index, which ranges from 0 to 8 . Scores from 5 to 8 indicate household food shortages which we utilized as our indicator of hunger-related stress $[44,45]$. The other constructs were created by standardizing and summing the items related to those constructs, then dichotomously scoring each variable to contrast the top quintile of each ("high stress") versus all others. An additional variable for the total count of high stress domains was created by summing these stress indicators, and categorizing the count as follows: 0, 1, 2, 3+. Measures of psychological distress, traumatic events, and chronic stressor domains can be found in Supplementary Material 1.

\section{Analyses}

First, we calculated descriptive statistics (mean and standard errors) for psychological distress, demographic, SES, and stressor variables, by race, and then by geographic type within the African population. To maintain the power of our 
analyses, we imputed missing values for all the variables included in the analyses using chained equations. When correctly implemented, multiple imputation procedures help to produce asymptotic unbiased estimates and standard errors [46]. The chained equation procedure allows us to impute multiple missing variables. We produced and performed analyses on 25 imputations of our dataset. While smaller numbers of imputations are commonly accepted for the validity of point estimates, a greater number of imputations gives us confidence in the replicability of our standard error estimates as well [47]. Then, using the imputed data, we weighted our dataset using Stata's svyset prefix based on the SANHANES study design and performed a series of multivariable linear regressions predicting psychological distress.

We utilized hierarchical linear regression models consistent with Pearlin's Stress Process framework to investigate predictors of psychological distress with the following analytic strategy: Model a estimated the association between distress and demographic variables (race, geographic locale, sex, and age). Model $b$ added variables for SES (education, household income, household wealth) to model a. Model c added a variable for the count of traumatic events to model b. Model d added a variable for a count of high chronic stress domains to model c. The fifth model, (model e), added the seven indicator variables for each of the chronic stressor domains simultaneously to model $\mathrm{c}$. The regression models were run on two samples: (1) the entire South African sample and (2) the African sub-sample.

\section{Results for South African population}

\section{Sample characteristics}

Table 1 presents the distributions of our main covariates and outcome variables in the sample population by race. Whites had the largest percentage of individuals with higher education, followed by Indian, Coloured, and African participants. A similar pattern is evident for the distribution of income and wealth. Africans had the highest percentage of individuals experiencing high stress in all the domains measured, except Crowding (Coloured, 23.1\%). Africans also had the highest proportion of individuals with 3 or more high stress domains $(24.2 \%)$, while Whites had the highest proportion of individuals reporting 4 or more traumatic events $(5 \%)$. Africans had the highest mean K-10 score of all the racial groups.

\section{Linear regression models for psychological distress}

Table 2 presents a series of linear regression models for psychological distress with each column representing a separate regression model. Model 1a, which includes demographic variables, indicates that Africans report higher distress than both White and Coloured participants. In Model 1b, with the inclusion of SES variables, disparities in psychological distress between the races remain significant, however, the disparity decreased between Africans and Whites by 55\%, and by $16 \%$ between African and Coloured participants. Across measures of SES, higher levels of education and wealth were associated with lower psychological distress.

In Model 1c, after including count of traumatic events, a significant disparity in psychological distress between Africans and Whites remains evident, with a reduction of only 3\%. Disparities between African and Coloured participants revealed a similar trend, falling 6\%. Education and the difference between the bottom and top quintiles of wealth remained significant, with little change in their coefficients. We also observed a graded association between the count of traumatic events and psychological distress.

In Model 1d, after adjusting for count of high chronic stress domains, disparities between African and White and Coloured participants remained significant but were reduced by $27 \%$ and $19 \%$, respectively. A graded association between the number of traumatic events and psychological distress persisted. Similarly, we observed a graded association between stressor count and psychological distress.

In Model 1e, hunger, interpersonal conflict, and neighborhood crime and alcohol-related offenses were associated with elevated psychological distress. Disparities between African and White participants were no longer significant; those between African and Coloured participants remained significant, but were reduced by $31 \%$.

\section{Results for the African population}

\section{Sample characteristics}

Table 3 presents the distributions of our main covariates and outcome variables among Africans by geographic locale. Africans living in formal urban settings had the largest percentage of individuals with higher education and in the top quintile of income and wealth. Prevalence of stressors varied by geographic locale. Africans living in formal rural settings had the highest proportion of individuals with 3 or more high chronic stress domains (37.7\%), while those living in formal urban settings had the highest proportion of individuals reporting 4 or more traumatic events $(4.5 \%)$. Africans living in formal urban settings had the highest mean K-10 score.

\section{Linear regression models for psychological distress}

Linear regression models were fit for psychological distress among Africans using the same hierarchical models described earlier, with a focus on geographic variation. 
Table 1 Descriptive statistics by race

\begin{tabular}{|c|c|c|c|c|c|c|c|c|c|c|c|c|c|}
\hline & \multicolumn{3}{|c|}{ African $(N=10,854)$} & \multicolumn{3}{|c|}{ White $(N=741)$} & \multicolumn{3}{|c|}{ Coloured $(N=3199)$} & \multicolumn{3}{|c|}{ Indian $(N=1383)$} & \multirow[t]{2}{*}{$p$ value* } \\
\hline & $N$ & $\%$ & SE & $N$ & $\%$ & SE & $N$ & $\%$ & SE & $N$ & $\%$ & SE & \\
\hline Age $<25$ & 3359 & $31 \%$ & $<0.01$ & 100 & $13.5 \%$ & 0.01 & 845 & $26.4 \%$ & 0.01 & 261 & $18.9 \%$ & 0.01 & $<0.01$ \\
\hline$>25$ and $<35$ & 2268 & $20.9 \%$ & $<0.01$ & 99 & $13.4 \%$ & 0.01 & 589 & $18.4 \%$ & 0.01 & 207 & $15 \%$ & 0.01 & \\
\hline$>35$ and $<45$ & 1666 & $15.4 \%$ & $<0.01$ & 148 & $2 \%$ & 0.01 & 561 & $17.6 \%$ & 0.01 & 253 & $18.3 \%$ & 0.01 & \\
\hline$>45$ and $<55$ & 1463 & $13.5 \%$ & $<0.01$ & 130 & $17.5 \%$ & 0.01 & 571 & $17.9 \%$ & 0.01 & 264 & $19.1 \%$ & 0.01 & \\
\hline$>55$ and $<65$ & 1104 & $10.2 \%$ & $<0.01$ & 145 & $19.6 \%$ & 0.01 & 377 & $11.8 \%$ & 0.01 & 236 & $17.1 \%$ & 0.01 & \\
\hline$>65$ & 985 & $9.1 \%$ & $<0.01$ & 119 & $16.1 \%$ & 0.01 & 253 & $7.9 \%$ & $<0.01$ & 162 & $11.7 \%$ & 0.01 & \\
\hline Female & 6429 & $59.3 \%$ & $<0.01$ & 395 & $53.5 \%$ & 0.02 & 1877 & $58.9 \%$ & 0.01 & 763 & $55.4 \%$ & 0.01 & $<0.01$ \\
\hline Male & 4412 & $40.7 \%$ & $<0.01$ & 343 & $46.5 \%$ & 0.02 & 1310 & $41.1 \%$ & 0.01 & 615 & $44.6 \%$ & 0.01 & \\
\hline No formal education & 2839 & $31.6 \%$ & $<0.01$ & 27 & $4.1 \%$ & 0.01 & 706 & $25.6 \%$ & 0.01 & 171 & $13.6 \%$ & 0.01 & $<0.01$ \\
\hline Grade $8-12$ & 5554 & $61.8 \%$ & 0.01 & 349 & $53.2 \%$ & 0.02 & 1860 & $67.5 \%$ & 0.01 & 873 & $69.4 \%$ & 0.01 & \\
\hline Higher education & 595 & $6.6 \%$ & $<0.01$ & 280 & $42.7 \%$ & 0.02 & 189 & $6.9 \%$ & $<0.01$ & 214 & $17 \%$ & 0.01 & \\
\hline Income $<$ R5000 & 3484 & $37.4 \%$ & 0.01 & 57 & $9.4 \%$ & 0.01 & 590 & $21 \%$ & 0.01 & 254 & $24.6 \%$ & 0.01 & $<0.01$ \\
\hline$>5000$ and $<10,000$ & 2017 & $21.7 \%$ & $<0.01$ & 25 & $4.1 \%$ & 0.01 & 557 & $19.9 \%$ & 0.01 & 92 & $8.9 \%$ & 0.01 & \\
\hline$>10,000$ and $<25,000$ & 2099 & $22.6 \%$ & $<0.01$ & 79 & $13 \%$ & 0.01 & 831 & $29.6 \%$ & 0.01 & 211 & $20.5 \%$ & 0.01 & \\
\hline$>25,000$ and $<50,000$ & 876 & $9.4 \%$ & $<0.01$ & 72 & $11.9 \%$ & 0.01 & 406 & $14.5 \%$ & 0.01 & 178 & $17.3 \%$ & 0.01 & \\
\hline$>50,000$ & 832 & $8.9 \%$ & $<0.01$ & 374 & $61.6 \%$ & 0.02 & 419 & $14.9 \%$ & 0.01 & 296 & $28.7 \%$ & 0.01 & \\
\hline Wealth Index Quintile 1 (Lowest) & 2519 & $27.9 \%$ & $<0.01$ & 8 & $1.3 \%$ & $<0.01$ & 256 & $9.8 \%$ & 0.01 & 1 & $0.1 \%$ & $<0.01$ & $<0.01$ \\
\hline 2 & 2362 & $26.2 \%$ & $<0.01$ & 12 & $1.9 \%$ & 0.01 & 309 & $11.8 \%$ & 0.01 & 40 & $3.4 \%$ & 0.01 & \\
\hline 3 & 1978 & $21.9 \%$ & $<0.01$ & 19 & $3.1 \%$ & 0.01 & 603 & $23 \%$ & 0.01 & 108 & $9 \%$ & 0.01 & \\
\hline 4 & 1405 & $15.6 \%$ & $<0.01$ & 97 & $15.7 \%$ & 0.01 & 820 & $31.3 \%$ & 0.01 & 303 & $25.4 \%$ & 0.01 & \\
\hline 5 & 758 & $8.4 \%$ & $<0.01$ & 482 & $78 \%$ & 0.02 & 630 & $24.1 \%$ & 0.01 & 742 & $62.1 \%$ & 0.01 & \\
\hline Low hunger & 6370 & $64.2 \%$ & $<0.01$ & 664 & $97.5 \%$ & 0.01 & 2385 & $84.6 \%$ & 0.01 & 1245 & $96.1 \%$ & 0.01 & $<0.01$ \\
\hline High hunger & 3557 & $35.8 \%$ & $<0.01$ & 17 & $2.5 \%$ & 0.01 & 434 & $15.4 \%$ & 0.01 & 50 & $3.9 \%$ & 0.01 & \\
\hline Low home alcohol stress & 7838 & $78.2 \%$ & $<0.01$ & 598 & $86.2 \%$ & 0.01 & 2361 & $82.3 \%$ & 0.01 & 1211 & $92.1 \%$ & 0.01 & $<0.01$ \\
\hline High home alcohol stress & 2189 & $21.8 \%$ & $<0.01$ & 96 & $13.8 \%$ & 0.01 & 509 & $17.7 \%$ & 0.01 & 104 & $7.9 \%$ & 0.01 & \\
\hline Low crowding & 7843 & $77.8 \%$ & $<0.01$ & 676 & $97.5 \%$ & 0.01 & 2233 & $76.9 \%$ & 0.01 & 1294 & $97.7 \%$ & $<0.01$ & $<0.01$ \\
\hline High crowding & 2239 & $22.2 \%$ & $<0.01$ & 17 & $2.5 \%$ & 0.01 & 672 & $23.1 \%$ & 0.01 & 31 & $2.3 \%$ & $<0.01$ & \\
\hline Low neighborhood inaccessibility & 7721 & $77.3 \%$ & $<0.01$ & 573 & $82.6 \%$ & 0.01 & 2424 & $84.8 \%$ & 0.01 & 1258 & $96.6 \%$ & 0.01 & $<0.01$ \\
\hline High neighborhood inaccessibility & 2266 & $22.7 \%$ & $<0.01$ & 121 & $17.4 \%$ & 0.01 & 434 & $15.2 \%$ & 0.01 & 44 & $3.4 \%$ & 0.01 & \\
\hline Low economic stress & 7648 & $78.8 \%$ & $<0.01$ & 614 & $93.5 \%$ & 0.01 & 2344 & $85.6 \%$ & 0.01 & 1026 & $84.1 \%$ & 0.01 & $<0.01$ \\
\hline High economic stress & 2059 & $21.2 \%$ & $<0.01$ & 43 & $6.5 \%$ & 0.01 & 394 & $14.4 \%$ & 0.01 & 194 & $15.9 \%$ & 0.01 & \\
\hline Low interpersonal conflict & 8035 & $79.7 \%$ & $<0.01$ & 576 & $80.8 \%$ & 0.01 & 2598 & $86.8 \%$ & 0.01 & 1084 & $84.6 \%$ & 0.01 & $<0.01$ \\
\hline High interpersonal conflict & 2048 & $20.3 \%$ & $<0.01$ & 137 & $19.2 \%$ & 0.01 & 395 & $13.2 \%$ & 0.01 & 198 & $15.4 \%$ & 0.01 & \\
\hline Low neighborhood crime and alcohol & 7575 & $77.2 \%$ & $<0.01$ & 657 & $95.4 \%$ & 0.01 & 2290 & $80.7 \%$ & 0.01 & 1135 & $87.6 \%$ & 0.01 & $<0.01$ \\
\hline High neighborhood crime and alcohol & 2243 & $22.8 \%$ & $<0.01$ & 32 & $4.6 \%$ & 0.01 & 547 & $19.3 \%$ & 0.01 & 160 & $12.4 \%$ & 0.01 & \\
\hline 0 stressors & 1538 & $18.5 \%$ & $<0.01$ & 275 & $48.5 \%$ & 0.02 & 786 & $33.2 \%$ & 0.01 & 578 & $57.1 \%$ & 0.02 & $<0.01$ \\
\hline 1 & 2513 & $30.2 \%$ & 0.01 & 217 & $38.3 \%$ & 0.02 & 793 & $33.5 \%$ & 0.01 & 305 & $30.1 \%$ & 0.01 & \\
\hline 2 & 2248 & $27.1 \%$ & $<0.01$ & 65 & $11.5 \%$ & 0.01 & 490 & $20.7 \%$ & 0.01 & 95 & $9.4 \%$ & 0.01 & \\
\hline $3+$ & 2010 & $24.2 \%$ & $<0.01$ & 10 & $1.8 \%$ & 0.01 & 300 & $12.7 \%$ & 0.01 & 34 & $3.4 \%$ & 0.01 & \\
\hline 0 Traumatic events & 8056 & $81.8 \%$ & $<0.01$ & 550 & $78.5 \%$ & 0.02 & 2443 & $83.1 \%$ & 0.01 & 1041 & $84.3 \%$ & 0.01 & $<0.01$ \\
\hline 1 & 607 & $6.2 \%$ & $<0.01$ & 53 & $7.6 \%$ & 0.01 & 224 & $7.6 \%$ & $<0.01$ & 86 & $7 \%$ & 0.01 & \\
\hline 2 & 467 & $4.7 \%$ & $<0.01$ & 36 & $5.1 \%$ & 0.01 & 138 & $4.7 \%$ & $<0.01$ & 50 & $4 \%$ & 0.01 & \\
\hline 3 & 330 & $3.4 \%$ & $<0.01$ & 27 & $3.9 \%$ & 0.01 & 76 & $2.6 \%$ & $<0.01$ & 32 & $2.6 \%$ & $<0.01$ & \\
\hline $4+$ & 385 & $3.9 \%$ & $<0.01$ & 35 & $5 \%$ & 0.01 & 59 & $2 \%$ & $<0.01$ & 26 & $2.1 \%$ & $<0.01$ & \\
\hline Kessler10 Score** & 9901 & 14.57 & 0.06 & 708 & 12.89 & 0.19 & 2957 & 13.17 & 0.1 & 1250 & 12.95 & 0.14 & $<0.01$ \\
\hline
\end{tabular}

Unweighted $N$ and weighted percentages

*Differences between multi-group comparisons using ANOVA and $\chi^{2}$ distribution

**Kessler10 Score values refer to mean and standard deviation 
Table 2 Linear regression models for psychological distress score $(N=15,981)$

\begin{tabular}{|c|c|c|c|c|c|}
\hline & $\begin{array}{l}1^{\mathrm{a}} \\
R^{2}=0.052 \\
\beta(\mathrm{SE})\end{array}$ & $\begin{array}{l}1^{\mathrm{b}} \\
R^{2}=0.065 \\
\beta(\mathrm{SE})\end{array}$ & $\begin{array}{l}1^{\mathrm{c}} \\
R^{2}=0.105 \\
\beta(\mathrm{SE})\end{array}$ & $\begin{array}{l}1^{* * * \mathrm{~d}} \\
R^{2}=0.139 \\
\beta(\mathrm{SE})\end{array}$ & $\begin{array}{l}1 * * * \mathrm{e} \\
R^{2}=0.251 \\
\beta(\mathrm{SE})\end{array}$ \\
\hline African & ref & $r e f$ & $r e f$ & $r e f$ & ref \\
\hline White & $-2.395(0.35)^{* *}$ & $-1.068(0.4)^{* *}$ & $-1.04(0.38)^{* *}$ & $-0.758(0.37)^{*}$ & $-0.564(0.31)$ \\
\hline Coloured & $-2.082(0.25)^{* *}$ & $-1.753(0.27) * *$ & $-1.649(0.25)^{* *}$ & $-1.336(0.24)^{* *}$ & $-0.923(0.21)^{* *}$ \\
\hline Indian & $-0.591(0.77)$ & $0.148(0.66)$ & $0.077(0.57)$ & $0.334(0.48)$ & $0.309(0.53)$ \\
\hline Female & ref & $r e f$ & $r e f$ & ref & $r e f$ \\
\hline Male & $-0.743(0.11)^{* *}$ & $-0.71(0.11)^{* *}$ & $-0.774(0.11)^{* *}$ & $-0.723(0.11)^{* *}$ & $-0.624(0.1)^{* *}$ \\
\hline Age & $0.056(<0.01)^{* *}$ & $0.048(<0.01)^{* *}$ & $0.043(<0.01)^{* *}$ & $0.045(<0.01)^{* *}$ & $0.029(<0.01)^{* *}$ \\
\hline No formal schooling & & ref & $r e f$ & $r e f$ & ref \\
\hline Grade $8-12$ & & $-0.813(0.19)^{* *}$ & $-0.849(0.19)^{* *}$ & $-0.628(0.18)^{* *}$ & $-0.482(0.17)^{* *}$ \\
\hline Higher education & & $-0.706(0.27)^{* *}$ & $-0.692(0.26)^{* *}$ & $-0.306(0.25)$ & $-0.37(0.24)$ \\
\hline Income $<$ R5000 & & ref & ref & ref & $r e f$ \\
\hline$>=5000 ;<10,000$ & & $0.177(0.25)$ & $0.172(0.24)$ & $0.152(0.23)$ & $0.146(0.21)$ \\
\hline$>=10,000 ;<25,000$ & & $-0.042(0.23)$ & $-0.065(0.23)$ & $-0.002(0.23)$ & $0.005(0.19)$ \\
\hline$>=25,000 ;<50,000$ & & $-0.124(0.27)$ & $-0.163(0.25)$ & $0.087(0.27)$ & $-0.063(0.25)$ \\
\hline$>=50,000$ & & $-0.437(0.23)$ & $-0.521(0.23)^{*}$ & $-0.229(0.22)$ & $-0.352(0.2)$ \\
\hline Wealth Index Quintile 1 (Lowest) & & $r e f$ & ref & $r e f$ & $r e f$ \\
\hline 2 & & $-0.191(0.27)$ & $-0.171(0.25)$ & $0.112(0.26)$ & $-0.21(0.22)$ \\
\hline 3 & & $0.203(0.31)$ & $0.19(0.29)$ & $0.542(0.31)$ & $0.158(0.25)$ \\
\hline 4 & & $-0.124(0.34)$ & $-0.11(0.32)$ & $0.465(0.34)$ & $-0.119(0.29)$ \\
\hline 5 & & $-1.185(0.4)^{* *}$ & $-1.083(0.36)^{* *}$ & $-0.23(0.39)$ & $-0.911(0.31)^{* *}$ \\
\hline 0 Traumatic events & & & ref & $r e f$ & ref \\
\hline 1 & & & $1.69(0.25)^{* *}$ & $1.581(0.24)^{* *}$ & $1.353(0.22)^{* *}$ \\
\hline 2 & & & $2.157(0.35)^{* *}$ & $1.991(0.34)^{* *}$ & $1.583(0.31)^{* *}$ \\
\hline 3 & & & $3.492(0.51)^{* *}$ & $3.188(0.48)^{* *}$ & $2.398(0.41)^{* *}$ \\
\hline $4+$ & & & $4.017(0.49)^{* *}$ & $3.752(0.47)^{* *}$ & $2.974(0.45)^{* *}$ \\
\hline 0 Stressors & & & & ref & \\
\hline 1 & & & & $1.302(0.19)^{* *}$ & \\
\hline 2 & & & & $2.079(0.22)^{* *}$ & \\
\hline $3+$ & & & & $3.328(0.3)^{* *}$ & \\
\hline Hunger & & & & & $0.698(0.17)^{* *}$ \\
\hline Home alcohol stress & & & & & $0.567(0.29)$ \\
\hline Crowding & & & & & $-0.325(0.18)$ \\
\hline Neighborhood inaccessibility & & & & & $-0.177(0.19)$ \\
\hline Economic stress & & & & & $0.021(0.21)$ \\
\hline Conflict & & & & & $5.383(0.26)^{* *}$ \\
\hline Neighborhood crime and alcohol & & & & & $0.523(0.21)^{*}$ \\
\hline
\end{tabular}

Data were analyzed on individuals aged 15 years and older who were interviewed. Therefore, data on 15,981 individuals of all race groups were analyzed for Table 2. The sample size presented in Table 2 is an unweighted $N$ and the regression analyses presented in Table 2 was conducted after multiple imputation

$* p<0.05$

$* * p<0.01$

***Model also adjusts for alcohol in home

${ }^{a}$ Demographic variables

${ }^{\mathrm{b}}$ Model 1a + SES variables

${ }^{\mathrm{c}}$ Model $1 \mathrm{~b}+$ traumatic events

${ }^{\mathrm{d}}$ Model $1 \mathrm{c}+$ stressor count

${ }^{\mathrm{e}}$ Model 1c+stressors 
Table 3 Descriptive statistics of African population by geographic locale

\begin{tabular}{|c|c|c|c|c|c|c|c|c|c|c|c|c|c|}
\hline & \multicolumn{3}{|c|}{$\begin{array}{l}\text { Formal urban } \\
(N=4207)\end{array}$} & \multicolumn{3}{|c|}{$\begin{array}{l}\text { Informal urban } \\
(N=1810)\end{array}$} & \multicolumn{3}{|c|}{$\begin{array}{l}\text { Informal rural [tribal] } \\
(N=3494)\end{array}$} & \multicolumn{3}{|c|}{$\begin{array}{l}\text { Formal rural [farms] } \\
(N=1343)\end{array}$} & \multirow[t]{2}{*}{$p$ value* } \\
\hline & $N$ & Mean & SE & $N$ & Mean & $\mathrm{SE}$ & $N$ & Mean & SE & $N$ & Mean & $\mathrm{SE}$ & \\
\hline Age $<25$ & 1221 & $29 \%$ & 0.01 & 579 & $32 \%$ & 0.01 & 1159 & $33.2 \%$ & 0.01 & 400 & $29.8 \%$ & 0.01 & $<0.01$ \\
\hline$>25$ and $<35$ & 875 & $20.8 \%$ & 0.01 & 476 & $26.3 \%$ & 0.01 & 608 & $17.4 \%$ & 0.01 & 309 & $23 \%$ & 0.01 & \\
\hline$>35$ and $<45$ & 664 & $15.8 \%$ & 0.01 & 345 & $19.1 \%$ & 0.01 & 436 & $12.5 \%$ & 0.01 & 221 & $16.5 \%$ & 0.01 & \\
\hline$>45$ and $<55$ & 671 & $16 \%$ & 0.01 & 217 & $12 \%$ & 0.01 & 393 & $11.3 \%$ & 0.01 & 182 & $13.6 \%$ & 0.01 & \\
\hline$>55$ and $<65$ & 433 & $10.3 \%$ & $<0.01$ & 121 & $6.7 \%$ & 0.01 & 417 & $12 \%$ & 0.01 & 133 & $9.9 \%$ & 0.01 & \\
\hline$>65$ & 342 & $8.1 \%$ & $<0.01$ & 71 & $3.9 \%$ & $<0.01$ & 476 & $13.6 \%$ & 0.01 & 96 & $7.2 \%$ & 0.01 & \\
\hline Female & 2449 & $58.3 \%$ & 0.01 & 1104 & $61 \%$ & 0.01 & 2134 & $61.2 \%$ & 0.01 & 742 & $55.3 \%$ & 0.01 & $<0.01$ \\
\hline Male & 1751 & $41.7 \%$ & 0.01 & 706 & $39 \%$ & 0.01 & 1355 & $38.8 \%$ & 0.01 & 600 & $44.7 \%$ & 0.01 & \\
\hline No formal education & 768 & $21.9 \%$ & 0.01 & 486 & $32.2 \%$ & 0.01 & 1039 & $36.1 \%$ & 0.01 & 546 & $50 \%$ & 0.02 & $<0.01$ \\
\hline Grade $8-12$ & 2325 & $66.2 \%$ & 0.01 & 993 & $65.7 \%$ & 0.01 & 1700 & $59.1 \%$ & 0.01 & 536 & $49.1 \%$ & 0.02 & \\
\hline Higher education & 417 & $11.9 \%$ & 0.01 & 32 & $2.1 \%$ & $<0.01$ & 137 & $4.8 \%$ & $<0.01$ & 9 & $0.8 \%$ & $<0.01$ & \\
\hline Income $<$ R5000 & 1111 & $30.7 \%$ & 0.01 & 601 & $39.4 \%$ & 0.01 & 1373 & $44.6 \%$ & 0.01 & 399 & $36.8 \%$ & 0.01 & $<0.01$ \\
\hline$>5000$ and $<10,000$ & 601 & $16.6 \%$ & 0.01 & 331 & $21.7 \%$ & 0.01 & 800 & $26 \%$ & 0.01 & 285 & $26.3 \%$ & 0.01 & \\
\hline$>10,000$ and $<25,000$ & 846 & $23.4 \%$ & 0.01 & 382 & $25.1 \%$ & 0.01 & 563 & $18.3 \%$ & 0.01 & 308 & $28.4 \%$ & 0.01 & \\
\hline$>25,000$ and $<50,000$ & 479 & $13.2 \%$ & 0.01 & 170 & $11.2 \%$ & 0.01 & 170 & $5.5 \%$ & $<0.01$ & 57 & $5.3 \%$ & 0.01 & \\
\hline$>50,000$ & 582 & $16.1 \%$ & 0.01 & 40 & $2.6 \%$ & $<0.01$ & 175 & $5.7 \%$ & $<0.01$ & 35 & $3.2 \%$ & 0.01 & \\
\hline Wealth Index Quintile 1 (Lowest) & 242 & $6.9 \%$ & $<0.01$ & 657 & $43.7 \%$ & 0.01 & 951 & $32.9 \%$ & 0.01 & 669 & $60.3 \%$ & 0.01 & $<0.01$ \\
\hline 2 & 561 & $15.9 \%$ & 0.01 & 419 & $27.9 \%$ & 0.01 & 1139 & $39.5 \%$ & 0.01 & 243 & $21.9 \%$ & 0.01 & \\
\hline 3 & 1029 & $29.2 \%$ & 0.01 & 266 & $17.7 \%$ & 0.01 & 540 & $18.7 \%$ & 0.01 & 143 & $12.9 \%$ & 0.01 & \\
\hline 4 & 990 & $28.1 \%$ & 0.01 & 145 & $9.6 \%$ & 0.01 & 229 & $7.9 \%$ & 0.01 & 41 & $3.7 \%$ & 0.01 & \\
\hline 5 & 701 & $19.9 \%$ & 0.01 & 16 & $1.1 \%$ & $<0.01$ & 28 & $1 \%$ & $<0.01$ & 13 & $1.2 \%$ & $<0.01$ & \\
\hline Low hunger & 2803 & $72.7 \%$ & 0.01 & 1026 & $61.8 \%$ & 0.01 & 1868 & $59.5 \%$ & 0.01 & 673 & $53.1 \%$ & 0.01 & $<0.01$ \\
\hline High hunger & 1055 & $27.3 \%$ & 0.01 & 633 & $38.2 \%$ & 0.01 & 1274 & $40.5 \%$ & 0.01 & 595 & $46.9 \%$ & 0.01 & \\
\hline Low home alcohol stress & 3031 & $77.9 \%$ & 0.01 & 1226 & $73.7 \%$ & 0.01 & 2622 & $81.9 \%$ & 0.01 & 959 & $75.6 \%$ & 0.01 & $<0.01$ \\
\hline High home alcohol stress & 861 & $22.1 \%$ & 0.01 & 438 & $26.3 \%$ & 0.01 & 580 & $18.1 \%$ & 0.01 & 310 & $24.4 \%$ & 0.01 & \\
\hline Low crowding & 3122 & $79.5 \%$ & 0.01 & 1197 & $70.8 \%$ & 0.01 & 2744 & $84.7 \%$ & 0.01 & 780 & $63.5 \%$ & 0.01 & $<0.01$ \\
\hline High crowding & 803 & $20.5 \%$ & 0.01 & 493 & $29.2 \%$ & 0.01 & 495 & $15.3 \%$ & 0.01 & 448 & $36.5 \%$ & 0.01 & \\
\hline Low neighborhood inaccessibility & 3525 & $91.9 \%$ & $<0.01$ & 1361 & $81 \%$ & 0.01 & 2297 & $71.4 \%$ & 0.01 & 538 & $42.8 \%$ & 0.01 & $<0.01$ \\
\hline High neighborhood inaccessibility & 309 & $8.1 \%$ & $<0.01$ & 319 & $19 \%$ & 0.01 & 920 & $28.6 \%$ & 0.01 & 718 & $57.2 \%$ & 0.01 & \\
\hline Low economic stress & 3112 & $83.3 \%$ & 0.01 & 1228 & $74.4 \%$ & 0.01 & 2317 & $74.8 \%$ & 0.01 & 991 & $81.2 \%$ & 0.01 & $<0.01$ \\
\hline High economic stress & 625 & $16.7 \%$ & 0.01 & 423 & $25.6 \%$ & 0.01 & 781 & $25.2 \%$ & 0.01 & 230 & $18.8 \%$ & 0.01 & \\
\hline Low interpersonal conflict & 3131 & $79.3 \%$ & 0.01 & 1413 & $82.2 \%$ & 0.01 & 2475 & $77.2 \%$ & 0.01 & 1016 & $83.9 \%$ & 0.01 & $<0.01$ \\
\hline High interpersonal conflict & 819 & $20.7 \%$ & 0.01 & 305 & $17.8 \%$ & 0.01 & 729 & $22.8 \%$ & 0.01 & 195 & $16.1 \%$ & 0.01 & \\
\hline Low neighborhood crime and alcohol & 2777 & $72.8 \%$ & 0.01 & 1079 & $65.4 \%$ & 0.01 & 2596 & $83.3 \%$ & 0.01 & 1123 & $90.9 \%$ & 0.01 & $<0.01$ \\
\hline High neighborhood crime and alcohol & 1039 & $27.2 \%$ & 0.01 & 571 & $34.6 \%$ & 0.01 & 520 & $16.7 \%$ & 0.01 & 113 & $9.1 \%$ & 0.01 & \\
\hline 0 Stressors & 798 & $24.9 \%$ & 0.01 & 199 & $13.5 \%$ & 0.01 & 422 & $16.2 \%$ & 0.01 & 119 & $11.5 \%$ & 0.01 & $<0.01$ \\
\hline 1 & 1015 & $31.7 \%$ & 0.01 & 417 & $28.4 \%$ & 0.01 & 823 & $31.6 \%$ & 0.01 & 258 & $24.9 \%$ & 0.01 & \\
\hline 2 & 857 & $26.8 \%$ & 0.01 & 392 & $26.7 \%$ & 0.01 & 729 & $28 \%$ & 0.01 & 270 & $26 \%$ & 0.01 & \\
\hline $3+$ & 529 & $16.5 \%$ & 0.01 & 461 & $31.4 \%$ & 0.01 & 629 & $24.2 \%$ & 0.01 & 391 & $37.7 \%$ & 0.02 & \\
\hline 0 Traumatic events & 3080 & $80.6 \%$ & 0.01 & 1375 & $81.4 \%$ & 0.01 & 2528 & $80.5 \%$ & 0.01 & 1073 & $89.9 \%$ & 0.01 & $<0.01$ \\
\hline 1 & 263 & $6.9 \%$ & $<0.01$ & 91 & $5.4 \%$ & 0.01 & 208 & $6.6 \%$ & $<0.01$ & 45 & $3.8 \%$ & 0.01 & \\
\hline 2 & 186 & $4.9 \%$ & $<0.01$ & 90 & $5.3 \%$ & 0.01 & 157 & $5 \%$ & $<0.01$ & 34 & $2.8 \%$ & $<0.01$ & \\
\hline 3 & 122 & $3.2 \%$ & $<0.01$ & 65 & $3.8 \%$ & $<0.01$ & 130 & $4.1 \%$ & $<0.01$ & 13 & $1.1 \%$ & $<0.01$ & \\
\hline $4+$ & 171 & $4.5 \%$ & $<0.01$ & 68 & $4 \%$ & $<0.01$ & 118 & $3.8 \%$ & $<0.01$ & 28 & $2.3 \%$ & $<0.01$ & \\
\hline Kessler10 Score** & 3886 & 14.82 & 0.1 & 1690 & 14.27 & 0.14 & 3134 & 14.68 & 0.11 & 1191 & 13.92 & 0.17 & $<0.01$ \\
\hline
\end{tabular}

Unweighted $N$ and Weighted Percentages

*Differences between multi-group comparisons using ANOVA and $\chi^{2}$ distribution

**Kessler10 Score values refer to mean and standard deviation 
Model 2a did not indicate any significant geographic disparities in psychological distress. In Model 2b, Africans living in formal urban settings reported significantly higher distress than those in the three other regions. Compared to the lowest quintile, those in the highest quintile of household income and wealth reported lower psychological distress. Educational attainment was inversely related to psychological distress.

In Model 2c, we did not observe any changes to the direction or significance of the results reported in Model $2 b$. Africans living in formal urban settings continued to report higher psychological distress than all other geographic locales. Those in the highest quintile of household income and wealth, as well as those who received a high school education or higher, continued to report lower psychological distress. The count of traumatic events demonstrated a graded positive association with psychological distress.

In Model 2d, disparities in psychological distress between Africans living in formal urban settings and those living in informal urban or rural settings were no longer significant. The psychological distress gap between Africans living in formal urban and rural settings decreased by $5 \%$. We also observed reductions in the magnitude of the association between SES and psychological distress. The differences in psychological distress between those in the lowest education category and those in the middle and highest remained significant but were reduced by $24 \%$ and $33 \%$, respectively. The difference in psychological distress between those in the bottom and the top quintile of wealth was reduced by $49 \%$ but remained significant. The difference between the bottom and top quintile in household income was reduced by $37 \%$ and was no longer significant. The counts of traumatic events and high chronic stress domains both displayed positive dose-response associations with psychological distress.

In Model 2e, hunger, interpersonal conflict, and stress related to problematic alcohol use in one's home were all positively associated with psychological distress. The psychological distress gap between Africans in formal urban and rural settings was reduced by $36 \%$ and was no longer significant. However, significant disparities in psychological distress emerged between Africans living in formal urban and informal rural locales, with higher psychological distress observed in the former (Table 4).

\section{Discussion}

The present study aimed to (1) assess the extent of racial disparities in psychological distress in a nationally representative sample, (2) explore the relationship between distress and the social conditions of life as captured by stressors and traumas, and (3) examine differences in psychological distress across formal/informal and urban/rural locales within the African subpopulation.

\section{Racial disparities in psychological distress}

The results provide invaluable insight into the status of mental health and its correlates within a nationally representative sample. We found that Africans had higher psychological distress than other racial groups, consistent with prior studies of race and mental health in South Africa [24, 25, 48]. We also found racial disparities in SES with Africans having the highest proportion of respondents with low education, income, and wealth. Racial disparities in psychological distress persisted after adjusting for SES, however, consideration of SES did reduce the magnitude of these disparities.

When including the count of high chronic stress domains in our model, we observed that wealth was no longer associated with lower psychological distress. However, when we examined the individual stress domains, wealth was again inversely associated with distress. This may suggest that wealth is not sufficiently protective when met with the cumulative burden of high-level stress across multiple domains.

Our findings concerning SES and racial disparities in psychological distress raise important questions. We do not know how well our indicators of SES captured the socioeconomic conditions we attempted to measure. Future research should aim to clarify whether our observed patterns are related to the measurement of SES. It is possible there exist other race-related aspects of economic hardship that are not captured in our measures. Further, there are race-related aspects of social experience, such as racial discrimination, that were not assessed in this study but which prior South African research indicated helps to explain the residual racial gap in psychological distress after adjusting for SES $[25,26]$.

\section{Cumulative burden of chronic stressors and traumatic events}

Roughly one-quarter of the study population experienced at least one lifetime traumatic event. Prior research has indicated a greater prevalence of traumatic events in South Africa; however, the measure we used contained fewer traumas than those previously utilized [24, 49]. The prevalence of stressors was much higher than that of traumatic events, with some 80 percent of respondents experiencing at least one.

We found that Africans reported the highest prevalence of traumatic events and at least one stressor. Africans were more likely to experience high chronic stress in all but one domain. Results from multiple regression models indicated a dose-response relationship between count of traumatic events and psychological distress, suggesting the cumulative 
Table 4 Linear regression models for psychological distress score among black South Africans $(N=10,723)$

\begin{tabular}{|c|c|c|c|c|c|}
\hline & $\begin{array}{l}2^{\mathrm{a}} \\
R^{2}=0.044 \\
\beta(\mathrm{SE})\end{array}$ & $\begin{array}{l}2^{\mathrm{b}} \\
R^{2}=0.063 \\
\beta(\mathrm{SE})\end{array}$ & $\begin{array}{l}2^{\mathrm{c}} \\
R^{2}=0.104 \\
\beta(\mathrm{SE})\end{array}$ & $\begin{array}{l}2 * * * \mathrm{~d} \\
R^{2}=0.131 \\
\beta(\mathrm{SE})\end{array}$ & $\begin{array}{l}2 * * * \mathrm{e} \\
R^{2}=0.246 \\
\beta(\mathrm{SE})\end{array}$ \\
\hline Formal urban & ref & ref & ref & ref & ref \\
\hline Informal urban & $-0.129(0.44)$ & $-0.947(0.47)^{*}$ & $-0.862(0.42)^{*}$ & $-0.769(0.41)$ & $-0.613(0.35)$ \\
\hline Informal rural (tribal) & $-0.099(0.34)$ & $-0.887(0.38)^{*}$ & $-0.781(0.34)^{*}$ & $-0.541(0.34)$ & $-0.628(0.28)^{*}$ \\
\hline Formal rural (farms) & $-0.642(0.54)$ & $-1.721(0.56)^{* *}$ & $-1.341(0.53)^{*}$ & $-1.27(0.54)^{*}$ & $-0.813(0.44)$ \\
\hline Female & ref & ref & ref & ref & ref \\
\hline Male & $-0.691(0.13)^{* *}$ & $-0.671(0.13)^{* *}$ & $-0.736(0.13)^{* *}$ & $-0.684(0.13)^{* *}$ & $-0.612(0.12)^{* *}$ \\
\hline Age & $0.067(<0.01)^{* *}$ & $0.058(<0.01)^{* *}$ & $0.052(<0.01)^{* *}$ & $0.054(<0.01)^{* *}$ & $0.036(<0.01)^{* *}$ \\
\hline No formal schooling & & ref & ref & ref & ref \\
\hline Grade $8-12$ & & $-0.851(0.21)^{* *}$ & $-0.846(0.21)^{* *}$ & $-0.647(0.2)^{* *}$ & $-0.465(0.18)^{*}$ \\
\hline Higher education & & $-1.122(0.31)^{* *}$ & $-1.096(0.3)^{* *}$ & $-0.732(0.3)^{*}$ & $-0.675(0.29)^{*}$ \\
\hline Income $<$ R5000 & & ref & $r e f$ & ref & $r e f$ \\
\hline$>=5000 ;<10,000$ & & $0.106(0.26)$ & $0.097(0.25)$ & $0.093(0.25)$ & $0.057(0.23)$ \\
\hline$>=10,000 ;<25,000$ & & $-0.105(0.27)$ & $-0.118(0.26)$ & $-0.005(0.26)$ & $-0.034(0.22)$ \\
\hline$>=25,000 ;<50,000$ & & $-0.183(0.32)$ & $-0.249(0.3)$ & $0.009(0.31)$ & $-0.194(0.29)$ \\
\hline$>=50,000$ & & $-0.722(0.28)^{*}$ & $-0.801(0.27)^{* *}$ & $-0.505(0.27)$ & $-0.613(0.24)^{*}$ \\
\hline Wealth Index Quintile 1 (Lowest) & & ref & ref & ref & ref \\
\hline 2 & & $-0.461(0.27)$ & $-0.393(0.26)$ & $-0.121(0.26)$ & $-0.363(0.22)$ \\
\hline 3 & & $-0.333(0.33)$ & $-0.269(0.32)$ & $0.113(0.32)$ & $-0.169(0.27)$ \\
\hline 4 & & $-0.683(0.38)$ & $-0.587(0.36)$ & $0.007(0.36)$ & $-0.484(0.3)$ \\
\hline 5 & & $-2.029(0.49)^{* *}$ & $-1.777(0.45)^{* *}$ & $-0.912(0.46)^{*}$ & $-1.398(0.38)^{* *}$ \\
\hline 0 traumatic events & & & $r e f$ & ref & ref \\
\hline 1 & & & $1.678(0.28)^{* *}$ & $1.561(0.29)^{* *}$ & $1.328(0.26)^{* *}$ \\
\hline 2 & & & $2.436(0.4)^{* *}$ & $2.278(0.39)^{* *}$ & $1.829(0.36)^{* *}$ \\
\hline 3 & & & $3.328(0.51)^{* *}$ & $3.061(0.49)^{* *}$ & $2.407(0.46)^{* *}$ \\
\hline $4+$ & & & $4.033(0.52)^{* *}$ & $3.824(0.51)^{* *}$ & $3.072(0.49)^{* *}$ \\
\hline 0 stressors & & & & ref & \\
\hline 1 & & & & $1.172(0.21)^{* *}$ & \\
\hline 2 & & & & $1.824(0.24)^{* *}$ & \\
\hline $3+$ & & & & $3(0.33)^{* *}$ & \\
\hline Hunger & & & & & $0.59(0.18)^{* *}$ \\
\hline Home alcohol stress & & & & & $0.728(0.23)^{* *}$ \\
\hline Crowding & & & & & $-0.332(0.2)$ \\
\hline Neighborhood inaccessibility & & & & & $-0.123(0.2)$ \\
\hline Economic stress & & & & & $-0.096(0.22)$ \\
\hline Conflict & & & & & $5.344(0.3)^{* *}$ \\
\hline Neighborhood crime and alcohol & & & & & $0.339(0.22)$ \\
\hline
\end{tabular}

Data were analyzed on individuals aged 15 years and older who were interviewed. Therefore, data on 10,723 Black African individuals were analyzed for Table 4 . The sample size presented in Table 4 is an unweighted $N$ and the regression analyses presented in Table 4 was conducted after multiple imputation

${ }^{*} p<0.05$

$* * p<0.01$

***Model also adjusts for alcohol in home

${ }^{a}$ Demographic variables

${ }^{\mathrm{b}}$ Model $2 \mathrm{a}+\mathrm{SES}$ variables

${ }^{\mathrm{c}}$ Model $2 \mathrm{~b}+$ traumatic events

${ }^{\mathrm{d}}$ Model $2 \mathrm{c}+$ stressor count

${ }^{\mathrm{e}}$ Model 2c+stressors 
burden of trauma is adversely affecting the mental health of South Africans. This is consistent with prior research in South Africa, the United States, and elsewhere [24, 50, 51]. We observed an analogous association between the count of high stress domains and psychological distress. To our knowledge, no other study has examined this relationship in South Africa; however, our results are consistent with literature in the United States, suggesting that the accumulation of stressors may be a marker of stress exposure severity and place a heavy burden on mental health [52-54].

We also investigated how these stressors and traumas explained existing racial disparities in psychological distress. After including the count of traumatic events and high chronic stress domains in our multivariable models, racial disparities in psychological distress between African and White, and African and Coloured individuals, decreased but remained significant. This suggests that the cumulative burden of high exposure to multiple stressors does not fully explain the disparity in psychological distress between Africans and Whites. However, our final model replaced count of high chronic stress domains with the individual chronic stressors and demonstrated that stress related to hunger, interpersonal conflict, neighborhood-level crime and alcohol abuse were significantly associated with psychological distress, and explained the gap in psychological distress between Africans and Whites. Previous studies reported that distress is explained by a combination of stressors and that no one measure in isolation is sufficient to explain it [48].

Our findings must be understood within the broader historical context of racism in South Africa. Our measures of stressors should be viewed as downstream effects of the oppressive systems engineered under Apartheid. For example, as a stressor, hunger encapsulates the physiological stress related to malnutrition as well as the culturally influenced psychological stress of failing to provide for one's family $[55,56]$. Hunger, particularly within this context, may also be considered as a marker of deprivation. That the multifaceted burden of hunger is differentially borne along racial lines may represent a long-term effect of policies designed to deprive Africans of material resources, including adequate income and good living conditions, manifesting itself in the current study as food-related and psychological disparities [57]. Similarly, neighborhood crime and alcohol abuse should be understood through a systemic lens. Alcohol has a long and insidious history of being used to maintain the imbalanced structure of power within colonial societies, particularly in South Africa, where farm laborers were often compensated with alcohol, and the effects thereof are still prevalent in recent times $[58,59]$. Alcohol has been used as a labor control device - a tool to obscure laborers' attention from their own pain and the working conditions that gave rise to it, and a deterrent against organization and protest [60-64]. From a systemic perspective, with diminished access to community-based mental health services, disadvantaged individuals are vulnerable to corporations' unregulated marketing of alcohol as a way to relieve emotional distress and suffering [65, 66]. Taken together, alcohol abuse itself, and its reciprocal relationship to neighborhood conditions and crime can be traced back to racially based deprivation. To develop policy-level and individual-level interventions to reduce racial disparities in psychological distress, it is important to recognize how colonial-era racially based deprivation, formalized into policy under Apartheid, can continue to drive the racial disparities in exposure to stressors documented in the present study.

\section{Geographic differences in psychological distress of Africans}

Another study aim was to investigate how psychological distress varied across geographic locations within the African subpopulation. Our results indicated Africans living in formal urban settings had higher psychological distress than those living in formal rural settings. We observed a stronger association between SES and psychological distress within the African subpopulation. This may indicate the emergence of class disparities among Africans as an additional determinant over and above racial disparities. Unlike the overall population, we observed a graded relation between education and psychological distress; for Africans, higher education was increasingly associated with lower psychological distress. Similarly, the wealthiest Africans reported lower psychological distress.

These results may appear counterintuitive-from an economic standpoint, Africans living in rural settings were generally more economically deprived, with less access to services and resources. The finding that Africans living in formal urban areas, that generally have more access to services and resources, had higher psychological distress is surprising and raises important questions about stressors that the African middle class may be exposed to. Historically, Apartheid-era geospatial planning ensured Africans were segregated, through the Group Areas Act of 1951 and forced removals, into more peripheral areas of towns and cities; and that "surplus labour" was corralled into so-called Bantustans, far away from urban Whites. These racial geospatial divisions have proved remarkably persistent in the 26 years since the end of Apartheid, and are significant determinants of risk factors to physical and mental health [67]. After the abolition of Apartheid in 1991, middle-class Africans gradually moved into historically White-occupied spacesspaces that are now formally open to them, but often remain majority White-occupied. Africans living alongside Whites in primarily higher SES environments, like the suburbs or some college campuses, likely represent a challenge to the status quo that was established by and has persisted since 
Apartheid. As a result, middle-class Africans may experience stress driven by experiences of discrimination and rejection $[68,69]$. For these Africans with higher SES, these experiences may also come in the primarily White workplace, which may be another source of stress [70].

Alternatively, the patterns noted for Africans living in formal urban settings could be a function of the heterogeneity of this group. While formal urban areas include suburbs (which are generally middle-to-high income, with some suburbs being very high income), this category also includes townships-formal high-density housing in urban areas that are not high income but can be middle and even low income, especially for African, Coloured or Indian individuals [71]. Thus, although formal urban areas generally have higher incomes and better access to services, as shown in Table 3 , some of the Africans in formal urban areas could be living in townships and could be exposed to other unmeasured stressors such as economic deprivation, fast-paced lifestyle, crowding, and other social stressors linked to urban living. Future research should better characterize the extent of heterogeneity of Africans in formal urban areas and the specific living and working conditions that they experience.

Our results offer important implications for policy development and provide insight into the ways equity and health may be intertwined. Policies aimed at making South Africa a healthier and more equitable society should focus on the nexus of these two constructs. Data on the efficacy of mental health interventions in South Africa remain relatively limited. A 2018 review of available treatments for common mental disorders evaluated with Randomized Controlled Trials indicated that since 2000 , only two interventions have been evaluated as treatments for anxiety, while eight were evaluated for depression [72]. Notably, one of the studies identified provided participants with access to small, individual loans. Men who were randomized to receive loans demonstrated lower depressive symptoms scores than those who did not, although this effect was not present among women [73]. Outside South Africa, existing research within the US has identified several different approaches for improving access to and efficacy of mental health treatment within racial minority groups, including hospital-based child wellness programs, linguistic/translational services, and community-based programs that foster positive psychological processes, including purpose and mastery [74-77].

However promising clinically focused initiatives may be, without also addressing the various structural factors which may perpetuate the accumulation of stressors and/ or traumatic events, efforts to reduce racial disparities in health are likely to fall short [14]. At the same time, policy changes implemented so far in South Africa may also be insufficient to alleviate racial disparities; despite Apartheid rule ending over 20 years ago, and over 300 of its policies being repealed, racial disparities in mental health persist as a result of the lingering pernicious effects of these policies [78]. The current racial geospatial landscape provides an excellent example of the above. The laws that implemented racial segregation by forced removals under the Group Areas Act disappeared in the late 1980s, and yet over 30 years later, the geospatial landscape still reflects the Apartheid heritage, restricting access to employment opportunities and mental health services, resulting in increased economic and psychological distress. While South Africa has been bold in its attempts to pass new policies to address the disparities created by Apartheid, existing research has identified several barriers which have slowed policy implementation, including as they relate to integrating mental health services into primary healthcare. Some of these barriers include the stigma of mental illness as a weakness, insufficiently trained nursing and mental health staff, and under-resourced health facilities in terms of staff, infrastructure, and medication [79-81]. For example, in an effort to provide equitable public healthcare to South Africans, the National Health Act was conceived in 2017, and yet the restructuring of the public health system for national health insurance has not yet occurred [82]. Current research on the struggle to provide equitable health services through national health insurance has indicated that a major implementation barrier rests in the disparity of human resources, including understaffing and inadequate management, between the private and public healthcare sectors, which serve, respectively, $18 \%$ and $82 \%$ of the population $[83,84]$. This then manifests itself as inequities in the provision of mental health services between these two sectors. As a result, those who are reliant on public healthcare, including most Africans, are not only more likely to suffer from psychological, social, and economic stressors, but also less likely to receive adequate treatment for them. Therefore, policy change to remove these barriers and improve access to treatment should be considered as approaches that can act in concert, by both changing the policies which have perpetuated racial disparities in mental health, while also alleviating the effects of such policies.

\section{Limitations and implications for future research}

In considering the findings of this study, several limitations are acknowledged. Since our data were cross-sectional, we lack information on the temporal ordering of our variables, and we can make no claims about causality. Furthermore, the data presented in this manuscript are almost a decade old and provide information about psychological distress, but not about mental disorders.

Despite the age of the data, our results are relevant to more recent South African history. In the past decade, South Africa has dealt with many societal and structural challenges that pose a risk for mental health. One such challenge has been persistent racial economic inequality-in 2008, on 
average, Africans earned 13 cents for every Rand of income Whites earned (when Apartheid fell, this gap was 12 cents for every Rand) [5, 85]. In 2018, this gap has narrowed but it is still striking with Africans earning 36 cents for every Rand of income Whites earned [86]. This economic inequity is critical to understanding the context of mental healthcare in South Africa. As described above, several barriers to the integration of mental health services into primary care facilities have created the conditions whereby there is extremely limited public access to mental health treatment outside of inpatient care. While private mental health resources exist, the cost of these services ensure that only wealthy South Africans (mostly White) can afford them. This reality suggests that persistent economic stressors-like hunger-will continue to be disproportionately experienced by Black and poor South Africans, and those who experience them will face barriers to receiving affordable treatment.

Our results are also germane to contemporary issues South Africa has faced during the COVID-19 pandemic. COVID-19, and the lockdown it has induced, has caused major disruptions in food supply chains partially due to lack of transportation and accessibility for farming communities [87]. Those South Africans who are insecure in their access to food and depend on social programs have seen cuts to these programs during the pandemic [88]. The lockdown also affected South Africa's struggles with alcohol abuse. After a controversial decision was made by public figures to ban alcohol products during the early stages of the pandemic, the South African healthcare system reported reductions in trauma cases, assault, accidents, sexual assault, and unnatural deaths [89-92].

Our results also have implications for the current political unrest South Africa is contending with, most of which has occurred within African and Indian townships. The rioting and subsequent looting that has taken place in these townships will have a marked negative effect on the psychological distress and economic stress of its residents. Furthermore, the violence that has occurred during the unrest (337 deaths as of July 22,2021) will have consequences for exposure to traumatic events at the population level and will also be disproportionately experienced by the majority African and Indian inhabitants of those townships [93, 94].

We therefore recommend that the SANHANES study be repeated, to determine the current status of mental health, social inequities in mental health, and the extent of progress in eliminating the racial disparities in psychological distress, especially in the wake of the COVID-19 pandemic and recent political unrest. We also note that SANHANES did not include any items measuring the stress of discrimination. Future nationally representative surveys should include measures of discrimination and other potential negative race-related experiences of racially disadvantaged South Africans at all SES levels [26]. Furthermore, while this analysis explored the extent to which contextual stressors accumulate, future research should aim to identify the extent of different patterns of stressor clustering and the degree to which such clusters may vary across different racial or socioeconomic groups.

These limitations notwithstanding, the results presented in this manuscript come from a nationally representative, probability sample of adult South Africans. Our findings provide the reader with invaluable insights into the state of racial disparities in mental health, the cumulative adverse associations of stressors and traumatic events with psychological distress, and how it varies by geographic locale within the African population.

\section{Conclusions}

The results of this scientific inquiry have implications for policy change and future research. We found racial disparities across several mental health-related domains. Africans had greater exposure to traumatic events, social stressors, and psychological distress. Within the African population, we observed disparities in psychological distress between urban and rural settings, with higher psychological distress among Africans in formal urban settings. Racial disparities in various domains of health are well documented in South Africa, and the results of this study contribute to an actionable knowledge base in this field. This research can also inform the development of policies that aim to make South Africa a healthier and more equitable society.

Supplementary Information The online version contains supplementary material available at https://doi.org/10.1007/s00127-021-02175-w.

Author contributions $\mathrm{NH}$ conducted statistical analyses and drafted the introduction, results, and discussion. DRW supervised data analysis and provided feedback on all sections of manuscript drafts. JWM conducted statistical analyses and drafted the methods and tables. RS, supervised data analysis, drafted the methods, and provided written feedback on the introduction, results, and discussion. TM, SS, and MM all provided written feedback on all sections of manuscript drafts. ADM and SPR developed the study design and provided written backback on all sections of manuscript drafts. All authors have read and agreed to the published version of the manuscript.

Funding Preparation of this paper was supported by the W.K. Kellogg Foundation, grant number P0131281.

Availability of data and materials Data will be made available upon request to the authors.

Code availability Data analysis code will be made available upon request to the authors. 


\section{Declarations}

Conflict of interest On behalf of all authors, the corresponding author states that there is no conflict of interest.

Ethical approval Ethical approval for the study was obtained from the Research Ethics Committee (REC) of the South African Human Sciences Research Council (HSRC) (REC number: 6/16/11/11). The Helsinki ethics protocol was followed throughout the course of this study.

Consent to participate/publication Informed written consent/assent to participate and publish was obtained from all the survey participants. Parental consent was also obtained from participants aged 15-17 years.

\section{References}

1. Stein DJ et al (2008) Lifetime prevalence of psychiatric disorders in South Africa. Br J Psychiatry 192(2):112-117

2. Jack $\mathrm{H}$ et al (2014) Closing the mental health treatment gap in South Africa: a review of costs and cost-effectiveness. Glob Health Action 7:23431

3. Herman AA et al (2009) The South African Stress and Health (SASH) study: 12-month and lifetime prevalence of common mental disorders. S Afr Med J = Suid-Afrikaanse tydskrif vir geneeskunde 99(5 Pt 2):339-344

4. Kessler RC et al (2009) The global burden of mental disorders: an update from the WHO World Mental Health (WMH) surveys. Epidemiol Psichiatr Soc 18(1):23-33

5. Leibbrandt M, Finn A, Woolard I (2012) Describing and decomposing post-apartheid income inequality in South Africa. Dev South Afr 29(1):19-34

6. Ataguba JE-O, Alaba O (2012) Explaining health inequalities in South Africa: a political economy perspective. Dev South Afr 29(5):756-764

7. Coovadia $\mathrm{H}$ et al (2009) The health and health system of South Africa: historical roots of current public health challenges. Lancet 374(9692):817-834

8. Francis D, Webster E (2019) Poverty and inequality in South Africa: critical reflections. Dev South Afr 36(6):788-802

9. Benatar SR (2013) The challenges of health disparities in South Africa. S Afr Med J 103:2013

10. Kon ZR, Lackan N (2008) Ethnic disparities in access to care in post-apartheid South Africa. Am J Public Health 98(12):2272-2277

11. Mayosi BM, Benatar SR (2014) Health and health care in South Africa-20 years after Mandela. N Engl J Med 371(14):1344-1353

12. The World Bank in South Africa - Overview. 2019, The World Bank.

13. Pearlin LI et al (1981) The stress process. J Health Soc Behav 22(4):337-356

14. Williams DR (2018) Stress and the mental health of populations of color: advancing our understanding of race-related stressors. J Health Soc Behav 59(4):466-485

15. Pearlin LI et al (2005) Stress, health, and the life course: some conceptual perspectives. J Health Soc Behav 46(2):205-219

16. Vega WA, Rumbaut RG (1991) Ethnic minorities and mental health. Ann Rev Sociol 17(1):351-383

17. Turner RJ (2013) Understanding health disparities: the relevance of the stress process model. Soc Mental Health 3(3):170-186

18. Andersen LS et al (2011) The psychometric properties of the K10 and K6 scales in screening for mood and anxiety disorders in the
South African Stress and Health study. Int J Methods Psychiatr Res 20(4):215-223

19. Kessler RC et al (2010) Screening for serious mental illness in the general population with the K6 screening scale: results from the WHO World Mental Health (WMH) survey initiative. Int J Methods Psychiatr Res 19(Suppl 1):4-22

20. Spies G et al (2009) Validity of the Kessler 10 (K-10) in detecting DSM-IV defined mood and anxiety disorders among pregnant women. Arch Womens Ment Health 12(2):69-74

21. Spies G et al (2009) Validity of the K-10 in detecting DSM-IVdefined depression and anxiety disorders among HIV-infected individuals. AIDS Care 21(9):1163-1168

22. Williams DR et al (2004) The South Africa stress and health study: rationale and design. Metab Brain Dis 19(1-2):135-147

23. Stein DJ, Williams DR, Kessler RC (2009) The South African Stress and Health (SASH) study: a scientific base for mental health policy. S Afr Med J 99(5 Pt 2):337

24. Williams SL et al (2007) Multiple traumatic events and psychological distress: the South Africa stress and health study. J Trauma Stress 20(5):845-855

25. Jackson PB et al (2010) Race and psychological distress: the South african stress and health study. J Health Soc Behav 51(4):458-477

26. Williams DR et al (2012) Perceived discrimination and psychological well-being in the U.S.A. and South Africa. Ethn Health 17(1-2):111-133

27. Pearlin LI, Schooler C (1978) The structure of coping. J Health Soc Behav 19(1):2-21

28. Mthembu JC et al (2017) Prevalence of psychological distress and its association with socio-demographic and HIV-risk factors in South Africa: findings of the 2012 HIV prevalence, incidence and behaviour survey. SSM Popul Health 3:658-662

29. Shisana O et al (2014) South African National HIV Prevalence, Incidence and Behaviour Survey

30. Maluleke T et al (2013) South African National Health and Nutrition Examination Survey (SANHANES-1)

31. Naidoo P et al (2018) The association between biopsychosocial factors and disability in a national health survey in South Africa. Psychol Health Med 23(6):653-660

32. Peltzer K, Pengpid S (2019) Prevalence, and social and health correlates of insomnia among persons 15 years and older in South Africa. S Afr J Psychol 49(4):467-478

33. Sewpaul R et al (2019) Psychological distress and C-reactive protein in a South African national survey. Acta Neuropsychiatr 31(5):270-275

34. McHiza ZJ et al (2019) Social and psychological predictors of body mass index among South Africans 15 years and older: SANHANES-1. Int J Environ Res Public Health 16(20):3919

35. Havenaar JM et al (2008) Common mental health problems in historically disadvantaged urban and rural communities in South Africa: prevalence and risk factors. Soc Psychiatry Psychiatr Epidemiol 43(3):209-215

36. Peer $\mathrm{N}$ et al (2013) Urban-rural and gender differences in tobacco and alcohol use, diet and physical activity among young black South Africans between 1998 and 2003. Glob Health Action 6:19216

37. Kessler RC et al (2002) Short screening scales to monitor population prevalences and trends in non-specific psychological distress. Psychol Med 32(6):959-976

38. Erasmus G (1991) Ian Goldin, Making Race: the politics and economics of Coloured identity in South Africa. London and New York: Longman, 1988, 312 pp., £13.50, ISBN 0582019796. Africa 61(4):559-561

39. Dwane N, Wabiri N, Manda S (2020) Small-area variation of cardiovascular diseases and select risk factors and their association to household and area poverty in South Africa: capturing emerging 
trends in South Africa to better target local level interventions. PLoS ONE 15(4):e0230564

40. McHiza ZJ et al (2020) Body image and the double burden of nutrition among South Africans from diverse sociodemographic backgrounds: SANHANES-1. Int J Environ Res Public Health 17(3):887

41. McHiza ZJ et al (2018) Understanding the determinants of hemoglobin and iron status: adolescent-adult women comparisons in SANHANES-1. Ann N Y Acad Sci. https://doi.org/10.1111/nyas. 13528

42. Berry KM et al (2017) Quantifying unmet need for hypertension care in South Africa through a care cascade: evidence from the SANHANES, 2011-2012. BMJ Glob Health 2(3):e000348

43. Gordon T, Booysen F, Mbonigaba J (2020) Socio-economic inequalities in the multiple dimensions of access to healthcare: the case of South Africa. BMC Public Health 20(1):289

44. Kleinman RE et al (1998) Hunger in children in the United States: potential behavioral and emotional correlates. Pediatrics 101(1):e3-e3

45. Labadarios D et al (2011) Food security in South Africa: a review of national surveys. Bull World Health Organ 89(12):891-899

46. White IR, Royston P, Wood AM (2011) Multiple imputation using chained equations: issues and guidance for practice. Stat Med 30(4):377-399

47. von Hippel PT (2020) How many imputations do you need? A two-stage calculation using a quadratic rule. Soc Methods Res 49(3):699-718

48. Turton RW, Chalmers BE (1990) Apartheid, stress and illness: the demographic context of distress reported by South African Africans. Soc Sci Med 31(11):1191-1200

49. Atwoli L et al (2013) Trauma and posttraumatic stress disorder in South Africa: analysis from the South African Stress and Health Study. BMC Psychiatry 13(1):182

50. Myers HF et al (2015) Cumulative burden of lifetime adversities: Trauma and mental health in low-SES African Americans and Latino/as. Psychol Trauma 7(3):243-251

51. Karam EG et al (2014) Cumulative traumas and risk threshoods: 12-month PTSD in the world mental health (WMH) surveys. Depress Anxiety 31(2):130-142

52. Thoits PA (2010) Stress and health: major findings and policy implications. J Health Soc Behav 51(Suppl):S41-53

53. McEwen BS (2006) Protective and damaging effects of stress mediators: central role of the brain. Dialogues Clin Neurosci 8(4):367-381

54. Sternthal MJ, Slopen N, Williams DR (2011) Racial disparities in health: how much does stress really matter? Du Bois Rev 8(1):95-113

55. Kruger LM, Lourens M (2016) Motherhood and the "Madness of Hunger": “...Want Almal Vra vir My vir 'n Stukkie Brood” (“... Because Everyone Asks Me for a Little Piece of Bread"). Cult Med Psychiatry 40(1):124-143

56. Chakona G, Shackleton CM (2017) Voices of the hungry: a qualitative measure of household food access and food insecurity in South Africa. Agric Food Secur 6(1):66

57. Pienaar L, von Fintel D (2014) Hunger in the former apartheid homelands: determinants of convergence one century after the 1913 land act. Agrekon 53(4):38-67

58. London L (1999) The "dop" system, alcohol abuse and social control amongst farm workers in South Africa: a public health challenge. Soc Sci Med 48(10):1407-1414

59. May PA et al (2019) The Dop System of Alcohol Distribution is Dead, but It's Legacy Lives On.... Int J Environ Res Public Health 16(19):3701

60. Frank JW, Moore RS, Ames GM (2000) Historical and cultural roots of drinking problems among American Indians. Am J Public Health 90(3):344-351
61. Curto JC (1989) Alcohol in Africa: a preliminary compilation of the post-1875 literature. Curr Bibliogr Afr Aff 21(1):3-31

62. Korieh CJ (2003) Alcohol and empire: "Illicit" gin prohibition and control in colonial Eastern Nigeria. Afr Econ Hist 31:111-134

63. Diduk S (1993) European alcohol, history, and the state in Cameroon. Afr Stud Rev 36(1):1-42

64. Willis J (2005) Drinking power: alcohol and history in Africa. History Compass. https://doi.org/10.1111/j.1478-0542.2005. 00176.x

65. Esser MB, Jernigan DH (2018) Policy approaches for regulating alcohol marketing in a global context: a public health perspective. Annu Rev Public Health 39(1):385-401

66. Morojele NK et al (2018) Alcohol marketing and adolescent alcohol consumption: results from the International Alcohol Control study (South Africa). S Afr Med J 108:2018

67. Mabin A (1992) Comprehensive segregation: the origins of the group areas act and its planning apparatuses. J South Afr Stud 18(2):405-429

68. Assari S, Moghani Lankarani M (2018) Workplace racial composition explains high perceived discrimination of high socioeconomic status African American Men. Brain Sci 8(8):139

69. Discrimination in America - Experiences and Views of African Americans, in Discrimination in America (2017) Robert Wood Johnson Foundation, NPR, Harvard T.H. Chan School of Public Health

70. Ren XS, Amick BC, Williams DR (1999) Racial/ethnic disparities in health: the interplay between discrimination and socioeconomic status. Ethn Dis 9(2):151-165

71. Census (2011) Census in brief 2012: Statistics South Africa

72. Kaminer D, Owen M, Schwartz B (2018) Systematic review of the evidence base for treatment of common mental disorders in South Africa. S Afr J Psychol 48(1):32-47

73. Fernald LC et al (2008) Small individual loans and mental health: a randomized controlled trial among South African adults. BMC Public Health 8:409

74. Williams DR, Cooper LA (2019) Reducing racial inequities in health: using what we already know to take action. Int J Environ Res Public Health 16(4):606

75. NeMoyer A et al (2020) Reducing racial/ethnic disparities in mental health service use among emerging adults: community-level supply factors. Ethn Health. https://doi.org/10.1080/13557858. 2020.1814999

76. Estes LJ, Savannah SB (2018) Catalyzing community action for mental health and wellbeing. Community Dev Invest Rev 1:21-31

77. Felzien M, Westfall JM, Zittleman L (2018) Building a mental, emotional, and behavioral health "community of solution" in Rural Colorado. Community Dev Invest Rev 1:81-90

78. Das-Munshi J et al (2016) Mental health inequalities in adolescents growing up in post-apartheid South Africa: cross-sectional survey, SHaW study. PLoS ONE 11(5):e0154478

79. Jacob N, Coetzee D (2018) Mental illness in the Western Cape Province, South Africa: a review of the burden of disease and healthcare interventions. SAMJ S Afr Med J 108:176-180

80. Bruwer B et al (2011) Barriers to mental health care and predictors of treatment dropout in the South African stress and health study. Psychiatr Serv 62(7):774-781

81. Petersen I, Lund C (2011) Mental health service delivery in South Africa from 2000 to 2010: one step forward, one step back. S Afr Med J 101(10):751-757

82. Docrat $\mathrm{S}$ et al (2019) Mental health system costs, resources and constraints in South Africa: a national survey. Health Policy Plan 34(9):706-719

83. Maphumulo WT, Bhengu BR (2019) Challenges of quality improvement in the healthcare of South Africa post-apartheid: a critical review. Curationis 42(1):e1-e9 
84. Mofolo N, Heunis C, Kigozi GN (2019) Towards national health insurance: alignment of strategic human resources in South Africa. Afr J Prim Health Care Fam Med 11(1):e1-e7

85. Leibbrandt $M$ et al (2010) Trends in South African Income Distribution and Poverty since the Fall of Apartheid

86. Sulla V, Zikhali P (2018) Overcoming Poverty and Inequality in South Africa: An Assessment of Drivers, Constraints and Opportunities

87. Arndt $\mathrm{C}$ et al (2020) Covid-19 lockdowns, income distribution, and food security: an analysis for South Africa. Glob Food Secur 26:100410

88. Ali Mohamed EM et al (2021) Food security and COVID-19 in Africa: implications and recommendations. Am J Trop Med Hyg 104(5):1613-1615

89. Banerjee I et al (2020) South Africa and its COVID-19 prohibition predilection. Nepal J Epidemiol 10(3):874-877
90. Reuter $\mathrm{H}$ et al (2020) Prohibiting alcohol sales during the coronavirus disease 2019 pandemic has positive effects on health services in South Africa. Afr J Prim Health Care Fam Med 12(1):e1-e4

91. Morris D et al (2020) Impact of lockdown measures implemented during the Covid-19 pandemic on the burden of trauma presentations to a regional emergency department in Kwa-Zulu Natal, South Africa. Afr J Emerg Med 10(4):193-196

92. Matzopoulos R et al (2020) South Africa's COVID-19 alcohol sales ban: the potential for better policy-making. Int J Health Policy Manag 9(11):486-487

93. Ni MY et al (2020) Mental health during and after protests, riots and revolutions: a systematic review. Aust N Z J Psychiatry 54(3):232-243

94. Unrest death toll rises to 337 (2021) Republic of South Africa: South African Government News Agency 